\title{
REVIEW
}

\section{Management of carcinoid syndrome: a systematic review and meta-analysis}

\author{
Johannes Hofland1', Aura D Herrera-Martínez1,2,3, Wouter T Zandee and Wouter W de Herder¹ \\ 'ENETS Center of Excellence, Section of Endocrinology, Department of Internal Medicine, Erasmus MC, Rotterdam, The Netherlands \\ 2Maimonides Institute for Biomedical Research of Cordoba (IMIBIC), Córdoba, Spain \\ ${ }^{3}$ Endocrinology and Nutrition Service, Reina Sofia University Hospital, Córdoba, Spain
}

Correspondence should be addressed to J Hofland: j.hofland@erasmusmc.nl

\begin{abstract}
Carcinoid syndrome (CS) is a debilitating disease caused by functional neuroendocrine tumors. Several treatment options are available to alleviate the hormonal symptoms, but their relative efficacy is unknown. Online databases were searched for publications on the treatment of CS symptoms. Independent reviewers assessed relevant publications for study quality and outcome. Meta-analysis of the outcomes of the intervention on CS-related symptoms was stratified by the type of treatment. We found 3682 therapeutic interventions on CS-specific outcomes were collected from 93 studies. Overall, the study qualities were poor with only six randomized controlled clinical trials. The somatostatin analogs octreotide and lanreotide induced symptomatic improvement in $65-72 \%$ and biochemical response in $45-46 \%$ of patients. An increase in dose or frequency or interclass switch led to a reduction of flushes and/or diarrhea in $72-84 \%$ of cases. Retrospective, institutional series showed that liver-directed therapy can improve symptoms in $82 \%$ of CS patients with a liver-dominant disease. The serotonin synthesis inhibitor telotristat ethyl reduced bowel movements in $40 \%$ of patients with diarrhea refractory to somatostatin analogs. Interferon-alpha controlled CS symptoms in 45-63\% of cases. Favorable response has been noted after radionuclide therapy in subgroup analyses of studies not specifically involving CS patients. Chemotherapy and everolimus did not induce a significant response in the CS. We conclude that several treatment lines can be offered to patients suffering from the carcinoid syndrome. Initiation of randomized controlled trials with a primary outcome on carcinoid syndrome symptoms is strongly recommended.
\end{abstract}

\author{
Key Words \\ - carcinoid syndrome \\ - somatostatin analogs \\ - diarrhea \\ - flushes \\ - 5-hydroxyindolacetic acid
}

\section{Introduction}

Neuroendocrine tumors (NETs) can produce a variety of biologically active substances that are capable of eliciting symptoms in patients (Hofland et al. 2018). The classical example of a hormonal syndrome caused by functional NETs is the carcinoid syndrome (CS). First described in 1931 (Scholte 1931), this syndrome is characterized by the occurrence of increased bowel movements, vasoactive flushes and bronchospasms as well as long-term fibrotic changes in the mesentery and of the cardiac valves (van der Lely \& de Herder 2005). The main mediator of carcinoid-related sequelae, particularly diarrhea and fibrosis, is thought to be the
C) 2019 Society for Endocrinology Published by Bioscientifica Ltd. Printed in Great Britain
Endocrine-Related Cancer (2019) 26, R145-R156 
amine derivative serotonin (5-hydroxytryptamine). Other co-secreted peptide hormones and amines include bradykinins, tachykinins and histamine and have also been implicated as causing cutaneous flushing and respiratory complaints in NET patients (Oates et al. 1964, Norheim et al. 1986).

CS is predominantly encountered in patients with midgut NETs, but can also be present in patients with NETs of other origin (Halperin et al. 2017). Importantly, the secreted vasoactive substances have to bypass the portal circulation and enter the systemic circulation directly since the liver will actively metabolize and inactivate these. This means that all serotonin in the portal vein is metabolized into the inactive 5-hydroxyindolacetid acid (5-HIAA). Gastroenteropancreatic NETs can metastasize to the liver, lymph nodes and bone, but CS is generally seen in patients with liver metastases (Grahame-Smith 1970). Consequently, the majority of CS patients cannot be treated with curative intention. The management of CS thus forms an important part of palliative care in this patient subset. Throughout the years, many different therapeutic modalities have been introduced for the treatment of CS, including liver-directed therapy, chemotherapy, interferonalpha (IFN), somatostatin analogs (SSAs), targeted radionuclides and drugs interfering with the serotonin pathway.

Treatment of individual patients should consider the patient's performance status and severity of complaints as well as tumor grade, stage and primary location. The international guidelines (Pavel et al. 2016, Strosberg et al. 2017b) recommend SSAs as firstline therapy in CS in stage IV NET patients. Second-line options include IFN, locoregional therapy, SSA dose increase, everolimus or peptide receptor radionuclide therapy (PRRT) using radiolabeled SSAs without a clear preference of one treatment above the other. This follows a general lack of availability of well-designed randomized clinical trials dedicated for the treatment of CS as well as the absence of guidelines for measuring CS symptom response in a standardized fashion. Three systematic reviews on the treatment of CS have been published that specifically focused on first-line SSAs (Modlin et al. 2010), high-dose SSAs (Broder et al. 2015) and refractory CS (Riechelmann et al. 2017). In order to advance the evidence-based treatment of CS, a systematic review and meta-analysis was undertaken to summarize available data on all known treatments of the hormonal complaints within the clinical spectrum of the CS.

\section{Methods}

\section{Literature search}

Online databases were mined on May 11, 2018, for publications on the treatment of carcinoid syndrome according to the following strategy: PubMed: ((()((malignant carcinoid syndrome[MeSH Terms] $)$ AND therapy [MeSH Terms]) AND english[Language])) NOT "review"[Publication Type])) NOT "case reports"[Publication Type]; Ovid: (carcinoid syndrome AND therapy NOT case report NOT review).af.; Web of Science: (carcinoid syndrome AND therapy) AND DOCUMENT TYPES: (Article) AND LANGUAGE: (English); Scopus: ( TITLE-ABS-KEY ( malignant AND carcinoid AND syndrome AND therapy ) AND LANGUAGE ( english ) ) AND DOCTYPE ( ar ) AND ( LIMIT-TO ( EXACTKEYWORD , "Human" ) ). This search yielded a total of 913 hits. Cochrane contained no published systematic reviews on CS, but only a single protocol (Guo et al. 2013). An additional group of 19 publications was added as crossreferences during the full-text screening.

\section{Selection of studies}

After the removal of duplicates, 820 publications were left for eligibility check. Through screening of the title and abstract, non-eligible studies were excluded. This left a selection of 157 publications for full-text evaluation. Because of the overall lack of randomized controlled trials, it was decided that retrospective series or singlearm treatment studies were also eligible for inclusion. Publications lacking a description of specific symptoms in the patient selection with CS or lacking quantitative outcome data were excluded. Consequently, studies only describing the antiproliferative effects of an intervention without symptom or biochemical data specifically in patients with CS were also excluded. This evaluation yielded a total of 92 publications that fitted in the inclusion criteria for meta-analysis. Evaluation was performed according to the Preferred Reporting Items for Systematic Reviews and Meta-analyses (PRISMA) statement (Moher et al. 2009). Figure 1 illustrates the study selection process.

\section{Data collection and risk of bias}

Full-text papers were scored independently by two authors ( $\mathrm{J} \mathrm{H}$ and A D H) using a standardized data extraction form, based on the Cochrane Guidelines (Higgins \& Green 2011). As an indicator for methodological quality, publications 


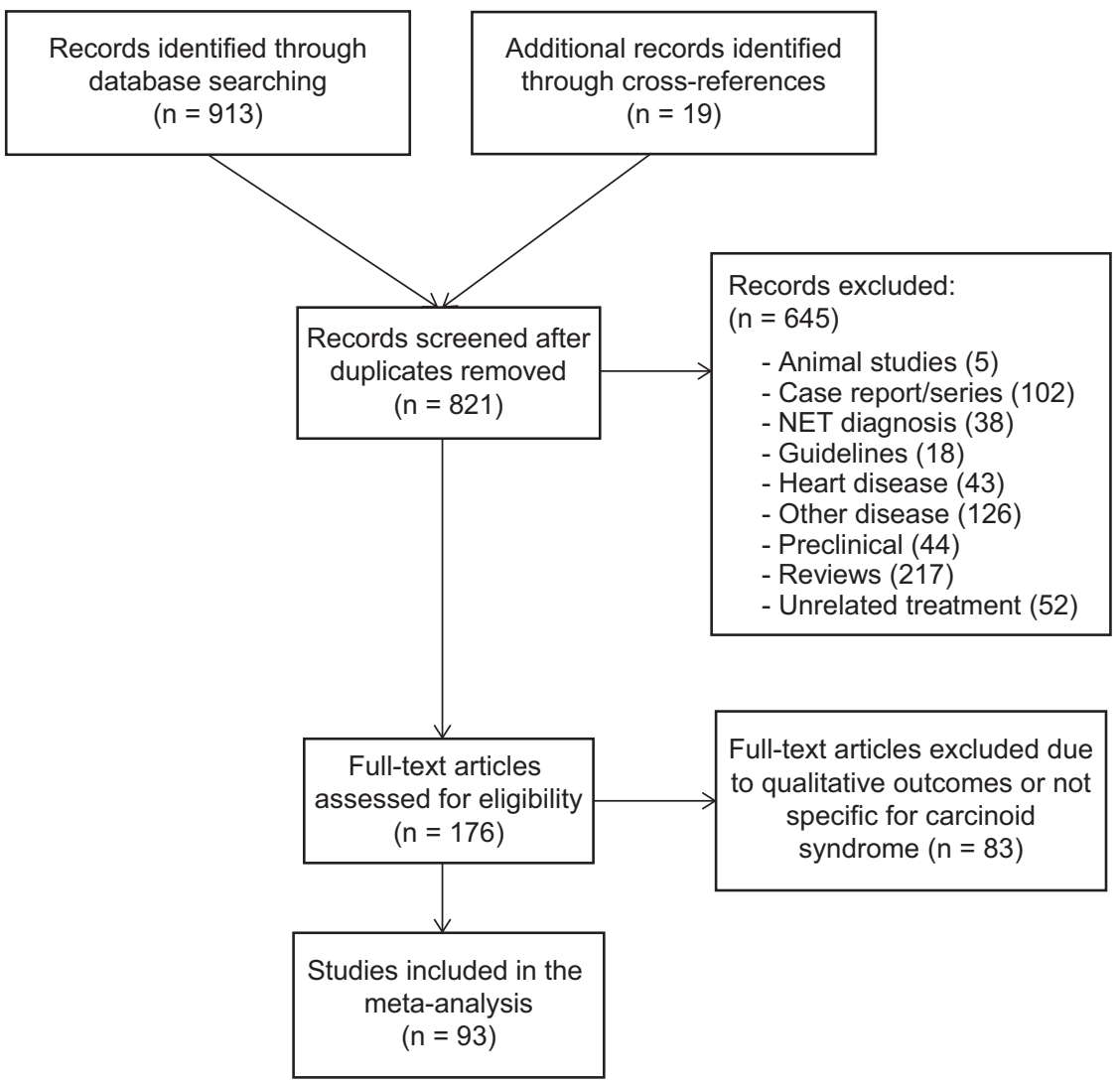

\section{Figure 1}

Flow diagram for study selection according to PRISMA guideline. were scored for several items for selection, performance and reporting bias using a low, medium or high scale denoting the quality of the publication. Selection bias was scored from the descriptions of randomization and inclusion criteria. Performance bias was evaluated by screening for descriptions of a control arm and allocation concealment. As criteria for reporting bias, incomplete data handling, selective reporting and industry funding were separately scored. Eligibility criteria and consistency in reporting were discussed at multiple teleconferences. Inconsistencies in scoring between the two reviewers were checked by a third author (W T Z) who made the final decision.

\section{Meta-analysis}

Data were constructed according to Cochrane Guidelines (Higgins \& Green 2011). Given the available treatment options, publications were allocated into one of the six groups: chemotherapy, IFN, SSA, liver-directed therapy, radionuclide therapy and serotonin pathway inhibitor. Also, data were extracted for a placebo-treated group. For the purpose of meta-analysis, efficacy outcome data were extracted as the percentage of patients with clinical or biochemical response following a specific intervention. Clinical response included overall symptom control and complaints of diarrhea and/or flushes. Biochemical response was evaluated as change in urinary 5-HIAA excretion specifically, and data on other biomarkers (such as chromogranin-A or gastrin) were excluded. Other (surrogate) outcomes such as stool consistency, bronchoconstriction, circulating serotonin levels or quality of life were recorded, but included insufficient data for meta-analysis. Duration of response was not recorded due to the overall inconsistent reporting of this outcome for symptom or biochemical control. Statistical analysis was performed using Prism, version 7 (Graphpad).

\section{Results}

\section{Quality check}

Overall, the methodological quality of the 93 studies that were selected was low, as summarized in Supplementary Table 1 (see section on supplementary data given at the end of this article). The presence of single-center retrospective series dominated over prospective studies and were at high risk of exaggerating the efficacy of
(C) 2019 Society for Endocrinology Published by Bioscientifica Ltd. Printed in Great Britain 
a given treatment modality in patients. However, the majority of prospective studies in CS patients also lacked a control arm and blinding of the patient and investigator. A considerable proportion of studies was not limited to CS patients, but also included patients with non-functional NETs or functional or syndromic pancreatic NETs. Only six studies focused on CS-specific outcomes and were designed in a randomized controlled fashion; all were industry funded and published after 2005 (Arnold et al. 2005, Pavel et al. 2011, 2018, Kulke et al. 2014, 2017, Vinik et al. 2016).

In total, 3682 individual therapeutic interventions in patients with CS were included in the meta-analysis.

\section{Outcome}

\section{Placebo}

Only four trials reporting on symptomatic or biochemical CS outcomes contained a placebo treatment arm (Supplementary Table 1). A total of 132 patients received placebo treatment in one study evaluating the SSA lanreotide (Vinik et al. 2016) and three studies in patients with refractory diarrhea to SSAs treated with telotristat ethyl (Kulke et al. 2014, 2017, Pavel et al. 2018). The effect of placebo was present but modest in these groups with 16 out of $56(29 \%)$ patients obtaining a symptomatic response and 9 out of $76(12 \%)$ patients with a reduction in diarrhea (Fig. 2). Additionally, 19\% of patients (11 out of 57) had a more than $30 \%$ decrease in their 5-HIAA levels.

\section{Somatostatin analogs}

Since the introduction of SSAs, different formulations of octreotide and lanreotide have been extensively studied in patients with CS. Both analogs predominantly target the somatostatin receptor subtype 2 (Hofland \& Lamberts 2003) and are regarded as first-line treatment for CS in unresectable and/or metastasized NET (Pavel et al. 2016, Strosberg et al. 2017b). More recently, the results of high-quality double-blind placebo-controlled trials studying the antiproliferative effects of octreotide LAR and lanreotide autogel led to the world-wide registration of these drugs as antiproliferative agents in NET patients. However, the PROMID study (Rinke et al. 2009) lacked data on symptomatic or biochemical control for a CS subset, whereas the CLARINET study (Caplin et al. 2014) did not include patients with the CS.

In this meta-analysis, 1945 interventions reported in 33 studies with these SSAs in CS patients have been collected (Supplementary Table 1). Many patients commencing on SSAs in these studies were pre-treated with different SSA formulations before enrolment (range 0-92\%). Six studies in 378 patients specifically investigated an escalation in SSA dose/frequency or SSA switch after progression under a regular dose of SSA. This included 72 patients who switched to the multi-somatostatin receptor-targeting analog pasireotide. These second-line treatment studies were evaluated separately.

After the start of therapy, octreotide induced a response of overall symptoms in $66 \%$, of diarrhea in $65 \%$ and of flushes in $72 \%$ of subjects (Fig. 2).

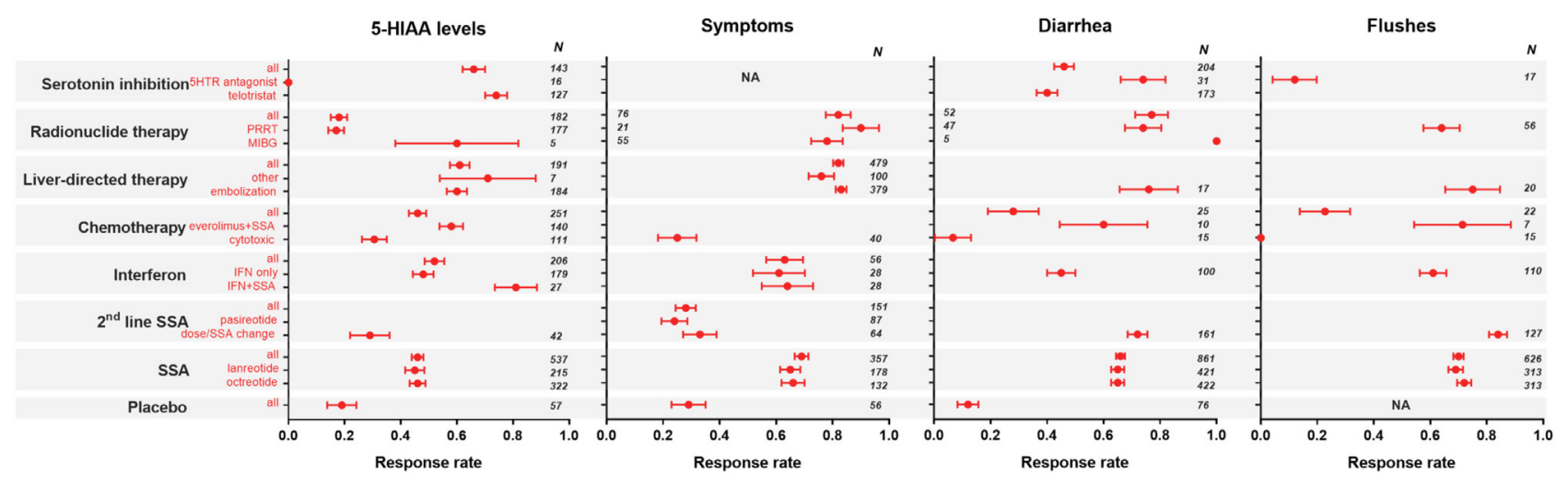

\section{Figure 2}

Meta-analysis of treatment outcomes for carcinoid syndrome-targeting therapies. Outcome of placebo and six separate intervention categories are depicted as mean (total) response rate \pm standard deviation. Definition of response differs between studies, but generally represents normalization or $>30-50 \%$ reduction in 5-HIAA levels or symptoms. The outcome of overall symptoms is dependent on the publication, but only descriptions specific for CS symptoms were included. All intervention categories are stratified according to dedicated studies as well as combined in 'all'. 5-HIAA, 5-hydroxyindolacetid acid; 5HTR, serotonin receptor; IFN, interferon-alpha; MIBG, 131/-metaiodobenzylguanidine; PRRT, peptide receptor radionuclide therapy with radiolabeled somatostatin analogs; SSA, somatostatin analog; N, number of patients in meta-analysis; NA, not available. 
Patients with the CS treated with lanreotide experienced similar response rates of 65,65 and $69 \%$, respectively. When combined with retrospective studies including the use of both analogs, control of the most relevant CS-elicited symptoms was obtained in $66-70 \%$ of patients. Conversely, the biochemical response of 5-HIAA levels was lower in $45-46 \%$ of CS patients treated with either SSA. Overall, effects were consistent between the different formulations (short vs long acting) or analogs (octreotide vs lanreotide) when evaluating control of the CS. The prospective studies that possessed a higher quality of design and large patient numbers reveal response rates that are compatible with these estimated percentages (Arnold et al. 1993, O'Toole et al. 2000, Pavel et al. 2011, Ruszniewski et al. 2016).

In the field of second-line treatment after SSA, two retrospective studies evaluated dose escalation (Strosberg et al. 2014, Al-Efraij et al. 2015), while two prospective studies investigated the effects of decreasing the injection interval to 21 days (Ferolla et al. 2012) or switching to the alternative SSA (O'Toole et al. 2000) (Supplementary Table 1). These strategies resulted in a reduction of diarrhea in 116 out of 161 (72\%) subjects and of flushes in 107 out of 127 (84\%) patients. The biochemical response reported in these studies was less pronounced at 29\% (Fig. 2). Alternatively, two studies in CS patients refractory to octreotide or lanreotide demonstrated that a SSA switch to pasireotide only led to a symptomatic response in 21 out of 87 (24\%) patients (Kvols et al. 2012, Wolin et al. 2013). Detailed information on diarrhea, flushes or 5-HIAA levels was lacking in refractory patients who were switched to pasireotide.

\section{Interferon-alpha}

Positive effects of IFN on carcinoid syndrome were first described in 1983 (Oberg et al. 1983). Studies in patients treated with IFN have focused on control of both proliferation as well as of CS symptoms. Importantly, fatigue, fever and flu-like symptoms occurring in the majority of patients has been described in most studies, hampering the tolerability of IFN (Oberg 2000).

Data on 347 patients from 16 studies treated with different formulations and combinations of IFNs were collected (Supplementary Table 1, see section on supplementary data given at the end of this article). A single RCT evaluated the effect of the addition of IFN to immediate-release octreotide (Arnold et al. 2005). In this multicenter study, 39 patients with CS were included and no significant additive effect of IFN was shown on top of the SSA when studying the CS-related outcomes. In four single-arm prospective series studying 73 CS patients, the reported response rates of IFN monotherapy varied widely between $0-90 \%$ and $50-80 \%$ for clinical and biochemical control, respectively (Moertel et al. 1989, Nobin et al. 1989, Veenhof et al. 1992, DiBartolomeo et al. 1996). Combining all IFN studies, including those with SSA or chemotherapy co-treatment, response rates for overall symptoms, diarrhea, flushes and 5-HIAA levels are 63, 45, 61 and $52 \%$, respectively (Fig. 2).

\section{Chemotherapy}

Studies evaluating the effect of chemotherapy on CS started as early as the 1960s. Most reports on chemotherapy for malignant CS are from before 2000, but were excluded in the current meta-analysis due to the inconsistent description of CS-specific outcomes. Unfortunately, most studies using chemotherapy restrict their data to the biochemical response (Supplementary Table 1). The reported 5-HIAA response rates following chemotherapeutical regimens including combinations of streptozotocin, cyclophosphamide, platinum derivatives or 5-fluoruracil are $31 \%$ in 111 patients in total (range 0-71\%). Clinical response data are limited to that for cisplatin $(0 \%)$, cyclophosphamide with methotrexate (6.7\%) and lomustine and 5-fluoruracil (60\%). Importantly, studies also detail about the significant adverse effect profiles observed in patients undergoing these various regimens.

Although not considered as chemotherapy, everolimus is registered as an antiproliferative treatment option for progressive NETs. In the RADIANT-2 trial (Pavel et al. 2011), the combination of octreotide LAR with everolimus elicited a biochemical response in in 81 out of 140 (61\%) patients with midgut NET and hormonal complaints, compared to 76 out of 141 (54\%) patients receiving octreotide LAR monotherapy. However, the prevalence of diarrhea was higher in the everolimus plus octreotide LAR group (27 vs 16\%). A small retrospective series (Bainbridge et al. 2015) showed improvement of diarrhea or flushes in 6 out of 10 and 5 out of 7 patients with refractory CS symptoms, respectively, after the addition of everolimus to octreotide.

\section{Liver-directed therapies}

This category is characterized by poor quality data, as evidenced by the many uncontrolled (100\%), retrospective (95\%), single institutional (100\%) series (Supplementary Table 1). Studies involved a multitude (c) 2019 Society for Endocrinology Published by Bioscientifica Ltd. Printed in Great Britain 
of interventional techniques including surgical segment resection, radiofrequency ablation, cryosurgery, bland embolization, chemoembolization or radioembolization. In the meta-analysis, hepatic interventions in 579 patients were collected from 25 studies. Often, patients were concurrently being treated with other modalities including SSAs, which presents a significant bias in interpretation of the outcome of liver-directed therapies. For most studies, the clinical response was described without further quantification or clarification of symptoms. Only 2 out of the 22 studies on liver-directed therapies stratified the changes in symptoms according to diarrhea or flushes (Maton et al. 1983, Drougas et al. 1998).

Overall reported clinical and biochemical response rates are high, especially in the very small series. The combination of the different liver-directed modalities resulted in a symptomatic response in 393 out of 479 (82\%) patients, whereas urinary 5-HIAA levels were significantly reduced in 116 out of 191 (61\%) patients (Fig. 2). The majority of studies concerned treatments with bland embolization, chemoembolization or radioembolization. When combining all embolization techniques, response rates are 82 and $63 \%$ for overall symptoms and serotonin release, respectively. Importantly, this efficacy does not appear to be further reduced in those patients who have been pre-treated with SSAs.

\section{Radionuclide therapy}

Systemic treatment of NETs with radiolabeled molecules has predominantly been given to patients with a progressive disease. Options include ${ }^{131}$ Iodine-labeled metaiodobenzylguanidine (131 I-MIBG) and ${ }^{90}$ Yttrium $\left({ }^{90} \mathrm{Y}\right)$ or ${ }^{177}$ Lutetium $\left({ }^{177} \mathrm{Lu}\right)$ labeled SSAs. Effects of ${ }^{131}$ I-MIBG on CS-specific outcomes have been described in four series, including one prospective study, in 48 patients in total (Table 1). Patients were pre-treated with SSAs in 13-100\% of cases. The meta-analysis for ${ }^{131}$ I-MIBG was restricted to the outcome of overall symptoms, which were alleviated in 43 out of 55 (78\%) patients (Fig. 2).

Peptide receptor radionuclide therapy (PRRT) with ${ }^{177} \mathrm{Lu}$-DOTATATE is an effective antiproliferative therapy in midgut NET patients progressing on a regular SSA dose (Strosberg et al. 2017a). Many institutional series have reported their experience with radiolabeled SSAs, but data specific to the treatment of CS is very limited. Through the systematic review, 156 patients with CS-specific outcomes after treatment with either ${ }^{177} \mathrm{Lu}$-DOTATATE or ${ }^{90}$ Y-DOTATOC were selected from four prospective phase 2 , single-center studies (Table 1). Overall biochemical response was poor in $17 \%$ of 177 patients available, but symptomatic improvements were more promising in $74 \%$ of 47 patients suffering from diarrhea or $64 \%$ of 56 patients with flushing (Fig. 2). These clinical effects were obtained despite a high prevalence of pre-treatment with non-radioactive SSAs (64-100\%).

\section{Serotonin pathway inhibitors}

As serotonin is a crucial contributor to the clinical complaints within the CS spectrum, studies have evaluated the inhibition of serotonin synthesis or action. The serotonin receptor antagonists cyproheptadine (Moertel et al. 1991) and ondansetron (Wymenga et al. 1998) have been evaluated in two prospective trials of patients with refractory CS. As expected, there was no biochemical response, but anti-diarrheal effects were seen in 6 out of 6 patients receiving ondansetron and in 6 out of 12 patients on cyproheptadine. A recent retrospective series confirmed the potency of ondansetron on refractory bowel symptoms in 11 out of 13 CS patients (Kiesewetter et al. 2018).

Recently, a novel serotonin synthesis inhibitor named telotristat ethyl was tested in a series of placebocontrolled, double-blind, phase 1-3, multicenter clinical trials in patients with CS refractory to SSAs. In the phase 1-2 studies (Pavel et al. 2011, Kulke et al. 2014), antidiarrheal and biochemical response was present in 33 and $31 \%$ of patients, respectively, on telotristat ethyl. The phase 3 trials which investigated the efficacy and safety of telotristat ethyl in patients with uncontrolled CS were designed for patients with either 4 or more stools per day (Kulke et al. 2017) or less than 4 stools per day (Pavel et al. 2018). Biochemical response was significantly higher in telotristat-treated patients compared to placebo treatment in both studies. A favorable effect of telotristat ethyl on diarrhea was shown in $40-44 \%$ of patients, compared to $0-20 \%$ of patients treated with placebo. In patients with 4 or more stools per day, placebo reduced daily stool frequency by 0.9 times, whereas telotristat ethyl $250 \mathrm{mg}$ tid or $500 \mathrm{mg}$ tid reduced daily stool frequency by 1.7 and 2.1 times, respectively. As expected from the known actions of serotonin, flushing was not significantly reduced.

\section{Discussion}

The carcinoid syndrome is a hormonal syndrome caused by the secretion of peptides and amines that stimulate gut motility, vasodilatation and fibrosis. Due to its rare (c) 2019 Society for Endocrinology Published by Bioscientifica Ltd. Printed in Great Britain 
incidence (Halperin et al. 2017), there is a lack of welldesigned clinical trials evaluating the efficacy and safety of surgical or medical interventions on CS-induced symptoms. This is illustrated by the first publication of a dedicated RCT for CS only 13 years ago (Arnold et al. 2005). Instead, most published data concern institutional experience, single-arm studies with limited patient numbers or subgroup analyses within larger studies incorporating non-functional NETs. This impedes the formulation of evidence-based decisions on the preferred therapy for patients with the CS. However, below we try to put the collected data into context and propose a strategy for CS patient management based on the available evidence. Importantly, we refrain from recommendations on the oncologic therapy of choice as this systematic review only evaluated the outcomes of symptoms or serotonin production. In the clinical setting, the hormonal syndrome should always be evaluated in conjunction with the tumor bulk, primary localization, tumor grade and stage, tumor growth rate and prior response to therapy as the available treatment options might have both antihormonal as well as antiproliferative outcomes. Also, consideration should be given to the wider differential diagnosis of diarrhea in NET patients, including infection, drug-induced diarrhea, steatorrhea due to exocrine pancreatic insufficiency, postoperative short bowel and bile malabsorption.

SSAs have been the principal CS treatment for decades. Despite the lack of randomized clinical trials at their introduction, evidence clearly supports their therapeutic efficacy in the majority of patients with CS. Our meta-analysis has shown clinical response rates in $65-70 \%$ of CS patients with equal efficacy of the two major SSAs octreotide and lanreotide. These findings are in line with the previously published meta-analysis from 2010 on SSAs in CS syndrome (Modlin et al. 2010), despite the addition of recent studies (Anthony \& Vinik 2011, Khan et al. 2011, Pavel et al. 2011, Martin-Richard et al. 2013, Ruszniewski et al. 2016, Vinik et al. 2016). Given their efficacy and favorable safety profile, octreotide and lanreotide constitute the preferred first-line therapies to control the hormonal sequelae of CS (Fig. 3).

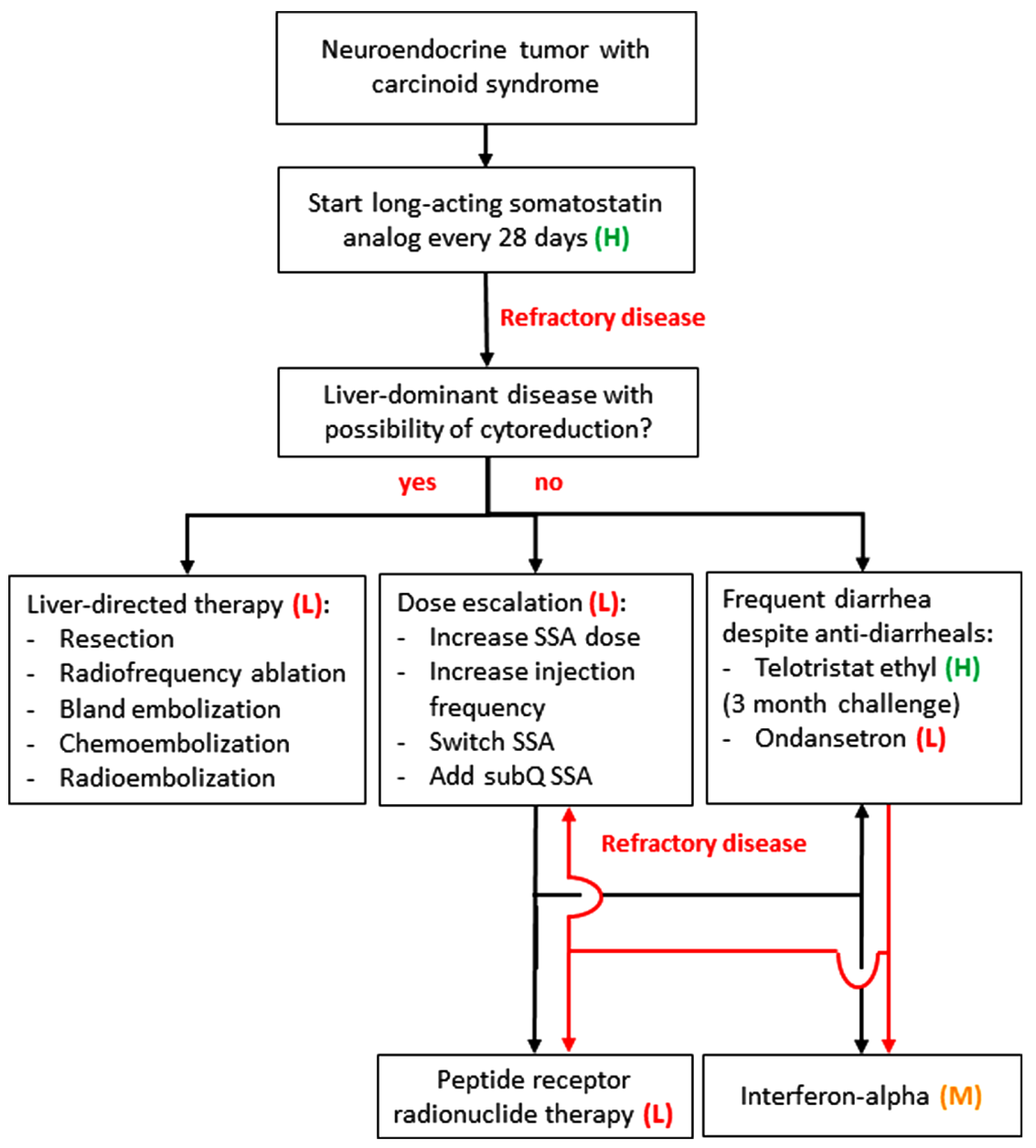

Figure 3

Proposed strategy for the management of carcinoid syndrome. Level of evidence is given according to GRADE approach (Atkins et al. 2004): low $(\mathrm{L})$, moderate $(\mathrm{M})$ or high $(\mathrm{H})$. 
There exists a patient preference for the long-acting monthly intramuscular or deep subcutaneous injections over the daily subcutaneous injections, which are equally potent (Rubin et al. 1999, O'Toole et al. 2000). Even in case of a potential curative resection of the (often metastasized) NET, preoperative initiation of SSAs should be considered obligatory in all symptomatic patients. Importantly, perioperative infusion with octreotide should also be commenced in symptomatic CS patients in order to prevent a carcinoid crisis (Woltering et al. 2016).

Multiple strategies have been investigated after clinical symptomatic progression during SSA therapy. As there is no reported upper limit of SSA dosing, studies have evaluated analog switch (O'Toole et al. 2000) or increments in injection frequency (Ferolla et al. 2012) or dose up to high or even ultra-high doses (Faiss et al. 1999, Welin et al. 2004, Strosberg et al. 2014, Al-Efraij et al. 2015). If tolerated, these therapeutic changes have the potential to control symptoms in up to $84 \%$ of patients without added toxicity and thus constitute a valuable alternative. Although not evaluated within a clinical trial, a subset of patients responds favorably to daily short-acting 'rescue' SSA injections on top of the long-acting SSA. Switching to the multireceptor-targeting pasireotide does not appear to have additional benefits. Clinical response rates of pasireotide therapy were similar to those observed in placebo-treated patients and accordingly are currently not advised for refractory CS patients.

As the hormonal efflux of CS predominantly originates from the liver and endocrine activity is correlated with tumor volume (Zandee et al. 2016), many centers have used surgical or embolization interventions to induce significant cytoreduction and consequently alleviate the hormonal symptoms. Despite the impressive response rates, the retrospective nature of 21 out of 22 studies renders a serious risk for reporting bias of successful cases. Although not part of the meta-analysis, peri-procedural complications and long-term adverse events such as liver failure should be taken into account. Current (peri-) procedural safety has advanced considerably from the early studies on hepatic artery ligation and embolization with 17-27\% mortality (Maton et al. 1983, Sjoblom et al. 1987). Consequently, liver-directed therapies could be considered in liver-dominant, refractory CS patients, depending on the liver anatomy and tumor localizations (Fig. 3). Options with extensive clinical experience include surgical resection, radiofrequency ablation, bland embolization and chemoembolization. A contemporary technique is selective internal radiation therapy through hepatic artery embolization with ${ }^{90} \mathrm{Y}$ - or ${ }^{166}$ Ho-labeled microparticles (Jia et al. 2017). Despite its limitations, meta-analysis was able to reveal no superiority in efficacy of one treatment over the other.

Following the pathophysiological relevance of serotonin in CS, several interventions in the serotonin pathway have been investigated. Serotonin receptors antagonists cyprohepatine (Moertel et al. 1991), ondansetron (Platt et al. 1992, Wymenga et al. 1998, Kiesewetter et al. 2018), ICS 205-930 (Coupe et al. 1988) and alosetron (Saslow et al. 1998) have been reported to confer favorable effects on bowel symptoms. However, the study quality was generally low in all, and 3 out of 6 studies could not be included in the meta-analysis due to a difference in outcome reporting. Medical inhibition of serotonin synthesis in CS patients has been reported as early as 1967 (Engelman et al. 1967), but clinical implementation has been restricted due to the adverse effects associated with cerebral serotonin deficiency. Recently, the oral serotonin synthesis inhibitor telotristat ethyl, which does not cross the blood-brain barrier, was introduced. In high-quality trials, telotristat ethyl inhibited diarrhea in CS patients with four or more bowel movements per day during SSA treatment. The anti-diarrheal effect of telotristat ethyl in patients with less than four bowel movements per day is statistically significant, but clinically limited at 0.4-0.6 less stools per day on average. Despite the high prevalence of biochemical response, clinical response was only obtained in $40 \%$ of patients. Consequently, a 3 -month trial of telotristat ethyl could be considered in CS patients with frequent diarrhea despite SSA (Joish et al. 2018). Telotristat ethyl should only be continued after the trial period in the case of a clear clinical benefit.

The first medical interventions for CS which were studied include chemotherapeutical regimens. Given that CS is predominantly encountered in well-differentiated, grade 1-2 NETs, chemotherapy has generally been found to exert little anti-tumoral effects. Consequently, the effects of chemotherapy on hormonal production and symptoms are disappointing at 46 and $25 \%$ of patients; the latter response rate approaches the level obtained in placebotreated patients. In the light of the severe adverse effects of the tested regimens, cytotoxic chemotherapy should be not administered to CS patients with well-differentiated NETs for purposes of hormonal control. Alternatively, everolimus has been reported to control CS-induced symptoms in case reports and series (Capdevila et al. 2011, Bainbridge et al. 2015). The additive effect on top on SSA was limited however in the randomized controlled
(C) 2019 Society for Endocrinology Published by Bioscientifica Ltd. Printed in Great Britain 
RADIANT-2 trial, and diarrhea is a known side effect of everolimus in 27-34\% of patients (Pavel et al. 2011, Yao et al. 2011, 2016).

Concomitantly with the advent of SSAs into clinical practice, IFN was also introduced into CS patients. IFN possesses antihormonal efficacy, although to a lesser extent that SSAs. Importantly, side effects including cytokine release preclude widespread use of this drug in CS patients. However, it is an alternative in patients refractory to SSAs, possibly after dose/frequency increment or after adding telotristat ethyl.

Finally, radionuclide therapy can be considered in refractory CS patients as a third-line option. Despite the potential bone marrow toxicity, nuclear therapy can evoke significant antihormonal response, presumably through cytoreduction. 131 I-MIBG was only tested in a small subset of CS patients, limiting the conclusions on its efficacy. PRRT with radiolabeled SSAs has extensively been studied in NET patients (Kwekkeboom et al. 2008) and is also registered for progressive midgut NETs after SSAs (Strosberg et al. 2017a), but to date, there are no dedicated studies for CS patients. Nonetheless, analyses of the CS subsets within the several prospective series did show promising results regarding its potent effect on hormonal symptoms in $82 \%$ of patients despite the high prevalence of SSA use.

In the light of the meta-analysis, we propose a management plan for patients with CS in Fig. 3, based on a GRADE approach for recommendations (Atkins et al. 2004). The favorable efficacy-to-toxicity profile of the SSAs octreotide and lanreotide secure them as firstline treatment of CS patients with an unresectable NET. Importantly, other non-hormonal causes of diarrhea and supportive measures such as anti-diarrheals should also be considered. In cases of CS that become refractory to first-line therapy, the option of significant hepatic cytoreduction through surgical resection or embolization is a viable strategy in selected patients with liver-dominant disease. When this is not feasible, escalation of the dose or frequency of the SSAs or an interclass switch could be considered. Alternatively, telotristat ethyl could be beneficial in a subset of patients with frequent diarrhea. Evidence for serotonin receptor antagonists is of poor quality, but could also be considered in refractory cases. These strategies have the potential to restrain CS in the majority of patients. However, for those cases with severe, refractory CS, clinicians could consider IFN or PRRT. The potential adverse effects and antiproliferative actions of individual treatment options should also be taken into consideration.
This study concerns the largest systematic review in the field of NET treatment and the first to evaluate all treatment modalities in CS through meta-analysis. Its strength is in the outcomes specific to CS symptoms and biochemistry, after exclusion of other hormonal syndromes, such as Zollinger-Ellison syndrome, insulinoma-induced organic hyperinsulinism or VIPomarelated diarrhea and flushing, and of other biomarkers, such as chromogranin-A or gastrin. Limitations include the overall poor quality of the included papers due to the lack of prospective trials within a dedicated group of CS patients. Data regarding quality of life, stool consistency, bronchospasm, fibrotic complications, adverse events or duration of response were too restricted or not systematically reported for inclusion into meta-analysis. Also, antiproliferative effects and progression-free survival were not included in order to focus completely on CS symptoms. Consistency in response rates throughout retrospective and prospective studies in the fields of SSA and IFN does allow the conclusion that these studies reliably estimate the treatment outcome.

There is a clear unmet need for further effective palliative treatment options for CS patients. The advent of telotristat ethyl holds promise for patients with persistent diarrhea, but only a minority of patients responded sufficiently and flushing complaints did not decrease. When studying the effects of drugs on CS, future trials should concentrate on the CS patient subset, provide clear descriptions of symptomatology before enrolment and study relevant patient outcomes. The more recent randomized controlled trials represent an appropriate model for this, but more investments should be made into the investigation of efficacy and safety of sequencing and combining existing treatments as well as of novel drug targets for CS. Learning from the past, one should study this in a dedicated, prospective, controlled trial within a clearly defined population of CS patients.

\section{Conclusions}

Efficacy of treatments targeting CS has predominantly been reported in studies of poor-to-moderate design. Despite this caveat, there are multiple treatment options to overcome hormonal production and complaints in the majority of patients. Following first-line therapy with SSAs, individual patient characteristics are the basis for decision making on how to go for several second- or third-line options with efficacy proven to a reasonable degree. 


\section{Supplementary data}

This is linked to the online version of the paper at https://doi.org/10.1530/ ERC-18-0495.

\section{Declaration of interest}

J $\mathrm{H}$ has received travel or speaker fees from Novartis, Ipsen and Advanced Accelerator Applications and is on the Advisory Board of Novartis. A D $\mathrm{H}$ and $\mathrm{W} T \mathrm{Z}$ have no potential conflict of interest. W W $\mathrm{H}$ has received travel or speaker fees from Novartis, Ipsen and Advanced Accelerator Applications, research funds from Ipsen and is on the Advisory Boards of Novartis and of Ipsen.

\section{Funding}

This work did not receive any specific grant from any funding agency in the public, commercial or not-for-profit sector.

\section{References}

Al-Efraij K, Aljama MA \& Kennecke HF 2015 Association of dose escalation of octreotide long-acting release on clinical symptoms and tumor markers and response among patients with neuroendocrine tumors. Cancer Medicine 4 864-870. (https://doi.org/10.1002/ cam4.435)

Anthony L \& Vinik AI 2011 Evaluating the characteristics and the management of patients with neuroendocrine tumors receiving octreotide LAR during a 6-year period. Pancreas 40 987-994. (https:// doi.org/10.1097/MPA.0b013e31821f66b4)

Arnold R, Neuhaus C, Benning R, Schwerk WB, Trautmann ME, Joseph K \& Bruns C 1993 Somatostatin analog sandostatin and inhibition of tumor growth in patients with metastatic endocrine gastroenteropancreatic tumors. World Journal of Surgery 17 511-519. (https://doi.org/10.1007/BF01655111)

Arnold R, Rinke A, Klose KJ, Muller HH, Wied M, Zamzow K, Schmidt C, Schade-Brittinger C, Barth P, Moll R, et al. 2005 Octreotide versus octreotide plus interferon-alpha in endocrine gastroenteropancreatic tumors: a randomized trial. Clinical Gastroenterology and Hepatology 3 761-771. (https://doi.org/10.1016/S1542-3565(05)00481-7)

Atkins D, Best D, Briss PA, Eccles M, Falck-Ytter Y, Flottorp S, Guyatt GH, Harbour RT, Haugh MC, Henry D, et al. 2004 Grading quality of evidence and strength of recommendations. BMJ $\mathbf{3 2 8}$ 1490. (https://doi.org/10.1136/bmj.328.7454.1490)

Bainbridge HE, Larbi E \& Middleton G 2015 Symptomatic control of neuroendocrine tumours with everolimus. Hormones and Cancer $\mathbf{6}$ 254-259. (https://doi.org/10.1007/s12672-015-0233-2)

Broder MS, Beenhouwer D, Strosberg JR, Neary MP \& Cherepanov D 2015 Gastrointestinal neuroendocrine tumors treated with high dose octreotide-LAR: a systematic literature review. World Journal of Gastroenterology 21 1945-1955. (https://doi.org/10.3748/wjg.v21. i6.1945)

Capdevila J, Diez Miranda I, Obiols G \& Tabernero J 2011 Control of carcinoid syndrome with everolimus. Annals of Oncology 22 237-239. (https://doi.org/10.1093/annonc/mdq670)

Caplin ME, Pavel M, Cwikla JB, Phan AT, Raderer M, Sedlackova E, Cadiot G, Wolin EM, Capdevila J, Wall L, et al. 2014 Lanreotide in metastatic enteropancreatic neuroendocrine tumors. New England Journal of Medicine 371 224-233. (https://doi.org/10.1056/ NEJMoa1316158)

Coupe MO, Anderson JV, Morris JA, Alstead EM, Bloom SR \& Hodgson HJ 1988 The effects of the 5-hydroxytryptamine (5HT3) receptor antagonist ICS 205-930 in the carcinoid syndrome.
Alimentary Pharmacology and Therapeutics 2 167-172. (https://doi. org/10.1111/j.1365-2036.1988.tb00684.x)

DiBartolomeo M, Bajetta E, Buzzoni R, Mariani L, Carnaghi C, Somma L, Zilembo N \& DiLeo A 1996 Clinical efficacy of octreotide in the treatment of metastatic neuroendocrine tumors - a study by the Italian Trials in Medical Oncology Group. Cancer 77 402-408. (https://doi.org/10.1002/(SICI)1097-0142(19960115)77:2<402::AIDCNCR25>3.0.CO;2-4)

Drougas JG, Anthony LB, Blair TK, Lopez RR, Wright JK, Jr, Chapman WC, Webb L, Mazer M, Meranze S \& Pinson CW 1998 Hepatic artery chemoembolization for management of patients with advanced metastatic carcinoid tumors. American Journal of Surgery 175 408-412. (https://doi.org/10.1016/S0002-9610(98)00042-7)

Engelman K, Lovenberg W \& Sjoerdsma A 1967 Inhibition of serotonin synthesis by para-chlorophenylalanine in patients with the carcinoid syndrome. New England Journal of Medicine 277 1103-1108. (https:// doi.org/10.1056/NEJM196711232772101)

Faiss S, Rath U, Mansmann U, Caird D, Clemens N, Riecken EO \& Wiedenmann B 1999 Ultra-high-dose lanreotide treatment in patients with metastatic neuroendocrine gastroenteropancreatic tumors. Digestion 60 469-476. (https://doi.org/10.1159/000007693)

Ferolla P, Faggiano A, Grimaldi F, Ferone D, Scarpelli G, Ramundo V, Severino R, Bellucci MC, Camera LM, Lombardi G, et al. 2012 Shortened interval of long-acting octreotide administration is effective in patients with well-differentiated neuroendocrine carcinomas in progression on standard doses. Journal of Endocrinological Investigation 35 326-331. (https://doi. org/10.3275/7869)

Grahame-Smith DG 1970 Progress report: the carcinoid syndrome. Gut 11 189-192. (https://doi.org/10.1136/gut.11.2.189)

Guo L, Wang P, Chen B \& Tang CW 2013 Somatostatin analogues for carcinoid syndrome (protocol). Cochrane Database of Systematic Reviews 2 CD010335. (https://doi.org/10.1002/14651858.CD010335)

Halperin DM, Shen C, Dasari A, Xu Y, Chu Y, Zhou S, Shih YT \& Yao JC 2017 Frequency of carcinoid syndrome at neuroendocrine tumour diagnosis: a population-based study. Lancet Oncology 18 525-534. (https://doi.org/10.1016/S1470-2045(17)30110-9)

Higgins JPT \& Green S 2011 Cochrane Handbook for Systematic Reviews of Interventions, 5.1.0 Version. London, UK: The Cochrane Collaboration. (available at: https://training.cochrane.org/handbook) Hofland LJ \& Lamberts SW 2003 The pathophysiological consequences of somatostatin receptor internalization and resistance. Endocrine Reviews 24 28-47. (https://doi.org/10.1210/er.2000-0001)

Hofland J, Zandee WT \& de Herder WW 2018 Role of biomarker tests for diagnosis of neuroendocrine tumours. Nature Reviews Endocrinology 14 656-669. (https://doi.org/10.1038/s41574-018-0082-5)

Jia ZZ, Paz-Fumagalli R, Frey G, Sella DM, McKinney JM \& Wang WP 2017 Single-institution experience of radioembolization with yttrium-90 microspheres for unresectable metastatic neuroendocrine liver tumors. Journal of Gastroenterology and Hepatology 32 1617-1623. (https://doi.org/10.1111/jgh.13752)

Joish VN, Frech F \& Lapuerta P 2018 Cost-effectiveness analysis of telotristat ethyl for treatment of carcinoid syndrome diarrhea inadequately controlled with somatostatin analogs. Journal of Medical Economics 21 182-188. (https://doi.org/10.1080/13696998.2017.1387 120)

Khan MS, El-Khouly F, Davies P, Toumpanakis C \& Caplin ME 2011 Long-term results of treatment of malignant carcinoid syndrome with prolonged release lanreotide (somatuline autogel). Alimentary Pharmacology and Therapeutics 34 235-242. (https://doi. org/10.1111/j.1365-2036.2011.04693.x)

Kiesewetter B, Duan H, Lamm W, Haug A, Riss P, Selberherr A, Scheuba C \& Raderer M 2018 Oral ondansetron offers effective antidiarrheal activity for carcinoid syndrome refractory to somatostatin analogs. Oncologist [epub]. (https://doi.org/10.1634/ theoncologist.2018-0191) (c) 2019 Society for Endocrinology Published by Bioscientifica Ltd. Printed in Great Britain 
Kulke MH, O'Dorisio T, Phan A, Bergsland E, Law L, Banks P, Freiman J, Frazier K, Jackson J, Yao JC, et al. 2014 Telotristat etiprate, a novel serotonin synthesis inhibitor, in patients with carcinoid syndrome and diarrhea not adequately controlled by octreotide. EndocrineRelated Cancer 21 705-714. (https://doi.org/10.1530/ERC-14-0173)

Kulke MH, Horsch D, Caplin ME, Anthony LB, Bergsland E, Oberg K, Welin S, Warner RRP, Lombard-Bohas C, Kunz PL, et al. 2017 Telotristat ethyl, a tryptophan hydroxylase inhibitor for the treatment of carcinoid syndrome. Journal of Clinical Oncology $\mathbf{3 5}$ 14-23. (https://doi.org/10.1200/JCO.2016.69.2780)

Kvols LK, Oberg KE, O'Dorisio TM, Mohideen P, de Herder WW, Arnold R, Hu K, Zhang Y, Hughes G, Anthony L, et al. 2012 Pasireotide (SOM230) shows efficacy and tolerability in the treatment of patients with advanced neuroendocrine tumors refractory or resistant to octreotide LAR: results from a phase II study. Endocrine-Related Cancer 19 657-666. (https://doi.org/10.1530/ ERC-11-0367)

Kwekkeboom DJ, de Herder WW, Kam BL, van Eijck CH, van Essen M, Kooij PP, Feelders RA, van Aken MO \& Krenning EP 2008 Treatment with the radiolabeled somatostatin analog (177 Lu-DOTA 0,Tyr3) octreotate: toxicity, efficacy, and survival. Journal of Clinical Oncology 26 2124-2130. (https://doi.org/10.1200/JCO.2007.15.2553)

Martin-Richard M, Massuti B, Pineda E, Alonso V, Marmol M, Castellano D, Fonseca E, Galan A, Llanos M, Sala MA, et al. 2013 Antiproliferative effects of lanreotide autogel in patients with progressive, well-differentiated neuroendocrine tumours: a Spanish, multicentre, open-label, single arm phase II study. BMC Cancer $\mathbf{1 3}$ 427. (https://doi.org/10.1186/1471-2407-13-427)

Maton PN, Camilleri M, Griffin G, Allison DJ, Hodgson HJ \& Chadwick VS 1983 Role of hepatic arterial embolisation in the carcinoid syndrome. BMJ 287 932-935. (https://doi.org/10.1136/ bmj.287.6397.932)

Modlin IM, Pavel M, Kidd M \& Gustafsson BI 2010 Review article: somatostatin analogues in the treatment of gastroenteropancreatic neuroendocrine (carcinoid) tumours. Alimentary Pharmacology and Therapeutics 31 169-188. (https://doi. org/10.1111/j.1365-2036.2009.04174.x)

Moertel CG, Rubin J \& Kvols LK 1989 Therapy of metastatic carcinoid tumor and the malignant carcinoid syndrome with recombinant leukocyte A interferon. Journal of Clinical Oncology 7 865-868. (https://doi.org/10.1200/JCO.1989.7.7.865)

Moertel CG, Kvols LK \& Rubin J 1991 A study of cyproheptadine in the treatment of metastatic carcinoid tumor and the malignant carcinoid syndrome. Cancer 67 33-36. (https://doi.org/10.1002/10970142(19910101)67:1<33::AID-CNCR2820670107>3.0.CO;2-E)

Moher D, Liberati A, Tetzlaff J, Altman DG \& PRISMA Group 2009 Preferred reporting items for systematic reviews and meta-analyses: the Prisma statement. BMJ 339 b2535. (https://doi.org/10.1136/bmj. b2535)

Nobin A, Lindblom A, Marnsson B \& Sundberg M 1989 Interferon treatment in patients with malignant carcinoids. Acta Oncologica 28 445-449. (https://doi.org/10.3109/02841868909111220)

Norheim I, Theodorsson-Norheim E, Brodin E \& Oberg K 1986 Tachykinins in carcinoid tumors: their use as a tumor marker and possible role in the carcinoid flush. Journal of Clinical Endocrinology and Metabolism 63 605-612. (https://doi.org/10.1210/jcem-63-3-605)

Oates JA, Melmon K, Sjoerdsma A, Gillespie L \& Mason DT 1964 Release of a kinin peptide in the carcinoid syndrome. Lancet 1 514-517. (https://doi.org/10.1016/S0140-6736(64)92907-1)

Oberg K 2000 Interferon in the management of neuroendocrine GEPtumors: a review. Digestion 62 (Supplement 1) 92-97. (https://doi. org $/ 10.1159 / 000051862)$

Oberg K, Funa K \& Alm G 1983 Effects of leukocyte interferon on clinical symptoms and hormone levels in patients with mid-gut carcinoid tumors and carcinoid syndrome. New England Journal of
Medicine 309 129-133. (https://doi.org/10.1056/ NEJM198307213090301)

O’Toole D, Ducreux M, Bommelaer G, Wemeau JL, Bouche O, Catus F, Blumberg J \& Ruszniewski P 2000 Treatment of carcinoid syndrome: a prospective crossover evaluation of lanreotide versus octreotide in terms of efficacy, patient acceptability, and tolerance. Cancer $\mathbf{8 8}$ 770-776. (https://doi.org/10.1002/(SICI)1097-

0142(20000215)88:4<770::AID-CNCR6>3.0.CO;2-0)

Pavel ME, Hainsworth JD, Baudin E, Peeters M, Horsch D, Winkler RE, Klimovsky J, Lebwohl D, Jehl V, Wolin EM, et al. 2011 Everolimus plus octreotide long-acting repeatable for the treatment of advanced neuroendocrine tumours associated with carcinoid syndrome (RADIANT-2): a randomised, placebo-controlled, phase 3 study. Lancet 378 2005-2012. (https://doi.org/10.1016/S01406736(11)61742-X)

Pavel M, O'Toole D, Costa F, Capdevila J, Gross D, Kianmanesh R, Krenning E, Knigge U, Salazar R, Pape UF, et al. 2016 Enets consensus guidelines update for the management of distant metastatic disease of intestinal, pancreatic, bronchial neuroendocrine neoplasms (NEN) and NEN of unknown primary site. Neuroendocrinology 103 172-185. (https://doi.org/10.1159/000443167)

Pavel M, Gross DJ, Benavent M, Perros P, Srirajaskanthan R, Warner RRP, Kulke MH, Anthony LB, Kunz PL, Horsch D, et al. 2018 Telotristat ethyl in carcinoid syndrome: safety and efficacy in the TELECAST phase 3 trial. Endocrine-Related Cancer 25 309-322. (https://doi. org/10.1530/ERC-17-0455)

Platt AJ, Heddle RM, Rake MO \& Smedley H 1992 Ondansetron in carcinoid syndrome. Lancet 339 1416. (https://doi.org/10.1016/01406736(92)91235-Z)

Riechelmann RP, Pereira AA, Rego JF \& Costa FP 2017 Refractory carcinoid syndrome: a review of treatment options. Therapeutic Advances in Medical Oncology 9 127-137. (https://doi. org/10.1177/1758834016675803)

Rinke A, Muller HH, Schade-Brittinger C, Klose KJ, Barth P, Wied M, Mayer C, Aminossadati B, Pape UF, Blaker M, et al. 2009 Placebocontrolled, double-blind, prospective, randomized study on the effect of octreotide LAR in the control of tumor growth in patients with metastatic neuroendocrine midgut tumors: a report from the PROMID Study Group. Journal of Clinical Oncology 27 4656-4663. (https://doi.org/10.1200/JCO.2009.22.8510)

Rubin J, Ajani J, Schirmer W, Venook AP, Bukowski R, Pommier R, Saltz L, Dandona P \& Anthony L 1999 Octreotide acetate long-acting formulation versus open-label subcutaneous octreotide acetate in malignant carcinoid syndrome. Journal of Clinical Oncology $\mathbf{1 7}$ 600-606. (https://doi.org/10.1200/JCO.1999.17.2.600)

Ruszniewski P, Valle JW, Lombard-Bohas C, Cuthbertson DJ, Perros P, Holubec L, Delle Fave G, Smith D, Niccoli P, Maisonobe P, et al. 2016 Patient-reported outcomes with lanreotide Autogel/Depot for carcinoid syndrome: an international observational study. Digestive and Liver Disease 48 552-558. (https://doi.org/10.1016/j. dld.2015.12.013)

Saslow SB, Scolapio JS, Camilleri M, Forstrom LA, Thomforde GM, Burton DD, Rubin J, Pitot HC \& Zinsmeister AR 1998 Medium-term effects of a new 5HT3 antagonist, alosetron, in patients with carcinoid diarrhoea. Gut 42 628-634. (https://doi.org/10.1136/ gut.42.5.628)

Scholte A 1931 Ein fall von angioma teleangiectaticum cutis mit chronischer endocarditis und malignem dünndarmcarcinoid. Beitrage Zur Pathologischen Anatomie 86 440-443.

Sjoblom SM, Hockerstedt K \& Jarvinen H 1987 Hepatic dearterialization in the treatment of carcinoid syndrome. Acta Chirurgica Scandinavica 153 523-529.

Strosberg JR, Benson AB, Huynh L, Duh MS, Goldman J, Sahai V, Rademaker AW \& Kulke MH 2014 Clinical benefits of above-standard dose of octreotide LAR in patients with neuroendocrine tumors for (c) 2019 Society for Endocrinology Published by Bioscientifica Ltd. Printed in Great Britain 
control of carcinoid syndrome symptoms: a multicenter retrospective chart review study. Oncologist 19 930-936. (https://doi.org/10.1634/ theoncologist.2014-0120)

Strosberg J, El-Haddad G, Wolin E, Hendifar A, Yao J, Chasen B, Mittra E, Kunz PL, Kulke MH, Jacene H, et al. 2017a Phase 3 trial of 177Lu-dotatate for midgut neuroendocrine tumors. New England Journal of Medicine 376 125-135. (https://doi.org/10.1056/ NEJMoa1607427)

Strosberg JR, Halfdanarson TR, Bellizzi AM, Chan JA, Dillon JS, Heaney AP, Kunz PL, O'Dorisio TM, Salem R, Segelov E, et al. 2017b The North American Neuroendocrine Tumor Society consensus guidelines for surveillance and medical management of midgut neuroendocrine tumors. Pancreas 46 707-714. (https://doi. org/10.1097/MPA.0000000000000850)

van der Lely AJ \& de Herder WW 2005 Carcinoid syndrome: diagnosis and medical management. Arquivos Brasileiros de Endocrinologia e Metabologia 49 850-860. (https://doi.org//S0004-27302005000500028)

Veenhof CHN, de Wit R, Taal BG, Dirix LY, Wagstaff J, Hensen A, Huldij AC \& Bakker PJM 1992 A dose-escalation study of recombinant interferon-alpha in patients with a metastatic carcinoid tumour. European Journal of Cancer 28 75-78. (https://doi. org/10.1016/0959-8049(92)90389-J)

Vinik AI, Wolin EM, Liyanage N, Gomez-Panzani E, Fisher GA \& ELECT Study Group 2016 Evaluation of lanreotide depot/autogel efficacy and safety as a carcinoid syndrome treatment (elect): a randomized, double-blind, placebo-controlled trial. Endocrine Practice 22 1068-1080. (https://doi.org/10.4158/EP151172.OR)

Welin SV, Janson ET, Sundin A, Stridsberg M, Lavenius E, Granberg D, Skogseid B, Oberg KE \& Eriksson BK 2004 High-dose treatment with a long-acting somatostatin analogue in patients with advanced midgut carcinoid tumours. European Journal of Endocrinology 151 107-112. (https://doi.org/10.1530/eje.0.1510107)
Wolin EM, Hu K, Hughes G, Bouillaud E, Giannone V \& Resendiz KH 2013 Safety, tolerability, pharmacokinetics, and pharmacodynamics of a long-acting release (LAR) formulation of pasireotide (SOM230) in patients with gastroenteropancreatic neuroendocrine tumors: results from a randomized, multicenter, open-label, phase I study. Cancer Chemotherapy and Pharmacology 72 387-395. (https://doi. org/10.1007/s00280-013-2202-1)

Woltering EA, Wright AE, Stevens MA, Wang YZ, Boudreaux JP, Mamikunian G, Riopelle JM \& Kaye AD 2016 Development of effective prophylaxis against intraoperative carcinoid crisis. Journal of Clinical Anesthesia 32 189-193. (https://doi.org/10.1016/j. jclinane.2016.03.008)

Wymenga ANM, de Vries EGE, Leijsma MK, Kema IP \& Kleibeuker JH 1998 Effects of ondansetron on gastrointestinal symptoms in carcinoid syndrome. European Journal of Cancer 34 1293-1294. (https://doi.org/10.1016/S0959-8049(98)00009-4)

Yao JC, Shah MH, Ito T, Bohas CL, Wolin EM, Van Cutsem E, Hobday TJ, Okusaka T, Capdevila J, de Vries EG, et al. 2011 Everolimus for advanced pancreatic neuroendocrine tumors. New England Journal of Medicine 364 514-523. (https://doi.org/10.1056/ NEJMoa1009290)

Yao JC, Fazio N, Singh S, Buzzoni R, Carnaghi C, Wolin E, Tomasek J, Raderer M, Lahner H, Voi M, et al. 2016 Everolimus for the treatment of advanced, non-functional neuroendocrine tumours of the lung or gastrointestinal tract (RADIANT-4): a randomised, placebo-controlled, phase 3 study. Lancet 387 968-977. (https://doi. org/10.1016/S0140-6736(15)00817-X)

Zandee WT, Kamp K, van Adrichem RC, Feelders RA \& de Herder WW 2016 Limited value for urinary 5-HIAA excretion as prognostic marker in gastrointestinal neuroendocrine tumours. European Journal of Endocrinology 175 361-366. (https://doi.org/10.1530/EJE16-0392)

Received in final form 2 January 2019

Accepted 3 January 2019

Accepted Preprint published online 3 January 2019 (c) 2019 Society for Endocrinology Published by Bioscientifica Ltd. Printed in Great Britain 